\title{
Long-Term Clinical Results and Management following Vitrectomy in Undetected Retinoblastoma Eyes
}

\author{
Eva Biewald ${ }^{a}$ Sabrina Schlüter ${ }^{a}$ Nikolaos E. Bechrakis ${ }^{a}$ Tobias Kiefer ${ }^{a}$ \\ Philipp Rating $^{\text {a }}$ Dirk Geismar ${ }^{b}$ Klaus A. Metz ${ }^{c}$ Sophia Göricke ${ }^{d}$ \\ Petra Ketteler ${ }^{\mathrm{e}}$ Norbert Bornfeld ${ }^{\mathrm{a}}$ \\ ${ }^{a}$ Department of Ophthalmology, University Hospital Essen, University Duisburg-Essen, Essen, Germany; ${ }^{b}$ West \\ German Proton Therapy Centre, University Hospital Essen, University Duisburg-Essen, Essen, Germany; ${ }^{C}$ Institute \\ of Pathology and Neuropathology, University Hospital Essen, University Duisburg-Essen, Essen, Germany; \\ ${ }^{\mathrm{d}}$ Department of Diagnostic and Interventional Radiology and Neuroradiology, University Hospital Essen, University \\ Duisburg-Essen, Essen, Germany; ${ }^{e}$ Department of Haematology/Oncology, University Children's Hospital Essen, \\ University Duisburg-Essen, Essen, Germany
}

\section{Keywords}

Masquerade syndrome · latrogenic tumour cell spread .

Adjuvant radiotherapy · Systemic chemotherapy ·

Metastatic disease $\cdot$ Enucleation

\begin{abstract}
Introduction: Given the rarity of retinoblastoma and the consequences of accidental vitrectomy in the event of misdiagnosis, reporting on clinical experience in this area is important. Objective: The aim of this study was to analyse the management and complications with a focus on local orbital recurrence and metastatic disease in 10 children vitrectomized in an undetected retinoblastoma eye. Methods: This is a retrospective descriptive case series conducted in a single-centre referral university hospital. Results: From October 1991 to June 2019, 10 patients with a vitrectomy in an unsuspected retinoblastoma eye were included in this study. The main preoperative diagnoses were unilateral inflammation with a suspected lymphoma, uveitis or toxocariasis in 5 cases, vitreous haemorrhage after trauma in 2 cases, and the last
\end{abstract}

3 were misdiagnosed with Coats disease, rhegmatogenous retinal detachment and congenital cataract. Mean age at surgery was 3 years, ranging from 14 months to 6 and a half years. Nine patients were suffering from unilateral retinoblastoma; these were enucleated and treated with 4-6 cycles of chemotherapy and/or radiation therapy. The sclerotomy sites were infiltrated with tumour cells in 3 cases. In 1 patient, the differential diagnosis of a malignant medulloepithelioma could not be excluded. One patient had bone marrow infiltration on initial presentation; all other patients are healthy without any signs of orbital recurrence or metastatic disease with a mean follow-up of 5.4 years. Conclusion: In children, intraocular tumours, including retinoblastoma and medulloepithelioma, should be ruled out before pars plana vitrectomy is performed. If no doubtless preoperative diagnosis can be established, preoperative magnetic resonance imaging is mandatory. If a vitrectomy in a retinoblastoma eye has been performed, immediate enucleation of the eye with subsequent chemotherapy and orbital radiation is effective to avoid local recurrence and systemic metastases.

(c) 2020 S. Karger AG, Basel karger@karger.com

(c) 2020 S. Karger AG, Basel

www.karger.com/oop

Karger"
Eva Biewald, MD

Department of Ophthalmology, University Hospital Essen Hufelandstrasse 55

DE-45147 Essen (Germany)

eva.biewald@uk-essen.de 


\section{Introduction}

Retinoblastoma is the most common intraocular malignancy worldwide but still a rare tumour with an estimated constant incidence of 1:16,000-18,000 live births each year [1]. The most recent 5-year survival rate in developed countries is as high as $96.5 \%$ due to advances in diagnosis and treatment options [2].

Reported risk factors for metastatic disease in retinoblastoma include optic nerve and intraneural invasion, choroidal invasion and transscleral migration to the orbit [3]. It is well known that extraocular involvement of any kind results in a considerable deterioration of the prognosis [4]. In particular, children with central nervous system (CNS) involvement tend to have an even worse prognosis than those with distant metastases in bones, bone marrow and lymph nodes without CNS involvement $[5,6]$.

Tumour cells of retinoblastoma may not only spread during the natural course of the disease but by surgical intraocular interventions, including fine-needle aspiration biopsy and vitrectomy. These procedures may result in intraorbital involvement and predisposition to extraocular extension of retinoblastoma with a high risk for systemic metastases and a high mortality rate [7].

It is widely accepted that a vitrectomy or biopsy in eyes with uncertain diagnosis in children should be avoided until the possibility of an underlying retinoblastoma has been excluded, as it can cause extraocular spread along the needle tract or vitrectomy sites [8-10].

In our paper, we discuss the long-term results, management and survival prognosis of inadvertently vitrectomized eyes in retinoblastoma patients.

\section{Material and Methods}

In this retrospective chart review, we included all children with a prior vitrectomy in an undetected retinoblastoma eye that were referred to our hospital from October 1991 to June 2019. We collected data on age, initial diagnosis, surgical procedure, laterality and time to referral to our clinic.

Data collection after the transfer to our hospital included staging, classification of the disease, histopathological features, patient management, treatment regimens and duration of follow up. Special emphasis was laid on metastatic spread and overall patient survival.

\section{Results}

In the period from 1991 to 2019 , we could include 10 patients with a history of a prior vitrectomy in an undetected retinoblastoma eye who were referred to our clinic.
Table 1. Patients' baseline characteristics, initial treatment and referral time

\begin{tabular}{|c|c|c|c|c|c|}
\hline $\begin{array}{l}\text { Patient } \\
\text { No. }\end{array}$ & $\begin{array}{l}\text { Age, } \\
\text { months }\end{array}$ & $\begin{array}{l}\text { Later- } \\
\text { ality }\end{array}$ & Initial diagnosis & $\begin{array}{l}\text { Initial } \\
\text { treatment }\end{array}$ & $\begin{array}{l}\text { Referral } \\
\text { time, } \\
\text { days }\end{array}$ \\
\hline 1 & 27 & U & Coats disease & $\mathrm{PPV} / \mathrm{PPL}$ & 62 \\
\hline 2 & 41 & $\mathrm{U}$ & haemorrhage & PPV/biopsy & 3 \\
\hline 3 & 79 & $\mathrm{U}$ & toxocariasis & PPV/biopsy & 4 \\
\hline 4 & 20 & $\mathrm{U}$ & inflammation & PPV/biopsy & 5 \\
\hline 5 & 14 & $\mathrm{U}$ & $\begin{array}{l}\text { retinal } \\
\text { detachment }\end{array}$ & PPV/biopsy & 10 \\
\hline 6 & 70 & $\mathrm{U}$ & endophthalmitis & PPV/biopsy & 14 \\
\hline 7 & 51 & $\mathrm{U}$ & inflammation & PPV/biopsy & 14 \\
\hline 8 & 19 & $\mathrm{U}$ & $\begin{array}{l}\text { congenital } \\
\text { cataract }\end{array}$ & PPL/biopsy & 20 \\
\hline 9 & 11 & $\mathrm{U}$ & uveitis & PPV/biopsy & 66 \\
\hline 10 & 21 & $\mathrm{U}$ & haemorrhage & $\mathrm{PPV} / \mathrm{PPL}$ & 7 \\
\hline
\end{tabular}

U, unilateral disease; PPV, pars plana vitrectomy; PPL, pars plana lentectomy.

All patients had unilateral disease. The mean age at vitrectomy was 36.1 months, with a range from 14 months to 6 and a half years.

The most frequent misdiagnosis resulting in an indication for vitrectomy was an unspecified unilateral inflammation with a presumed diagnosis of uveitis, toxocariasis or endophthalmitis in 5 cases. Two children were treated due to a presumed vitreous haemorrhage after a blunt ocular trauma, 1 child was misdiagnosed with Coats disease, and the 2 remaining children were treated for rhegmatogenous retinal detachment or congenital cataract. Nine patients had a complete vitrectomy; 1 child was treated with pars plana lentectomy and tumour biopsy after the initial misdiagnosis of a congenital cataract. The mean time interval between vitrectomy and referral to our institution was 21 days on average, ranging from 3 days to 2 months in 2 cases. The delay in the referral of these 2 children was due to an inconclusive vitrectomy sample in 1 case, which could only be clarified after secondary enucleation elsewhere, and difficulties in obtaining a visa to Europe in the other case. Table 1 gives an overview.

On referral to our institution, all patients were examined carefully, and the diagnosis of retinoblastoma could be established in 7 out of the remaining 8 eyes. Two patients had already been enucleated elsewhere, leading to the final diagnosis of a retinoblastoma.

The eyes were staged according to the International Intraocular Retinoblastoma Classification (IIRC). Six of 

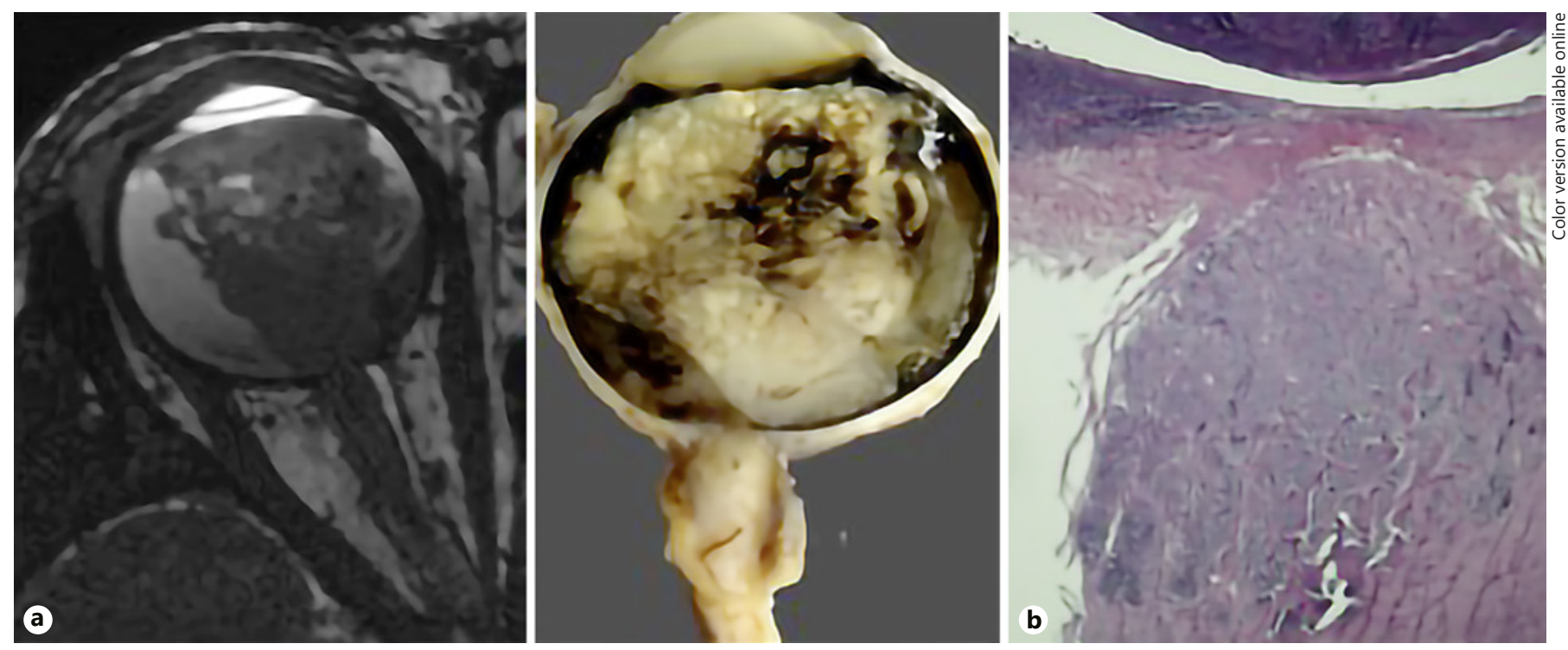

Fig. 1. Corresponding MRI, macroscopic photography and histopathological slide of patient No. 7 with 5-mm optic nerve infiltration.

2

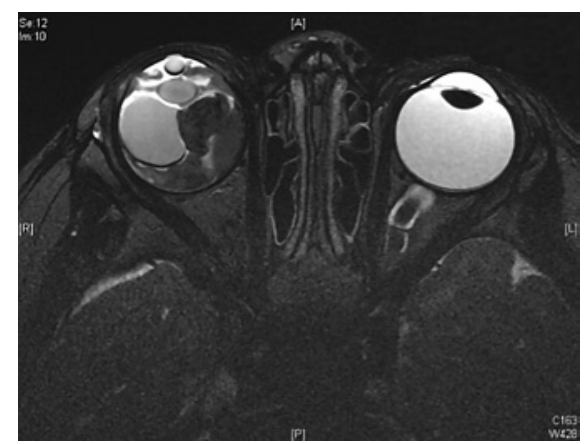

Fig. 2. MRI scan of patient No. 9 with extraocular tumour growth nasal to the optic nerve and silicone oil tamponade after $25-\mathrm{G}$ vitrectomy.
3
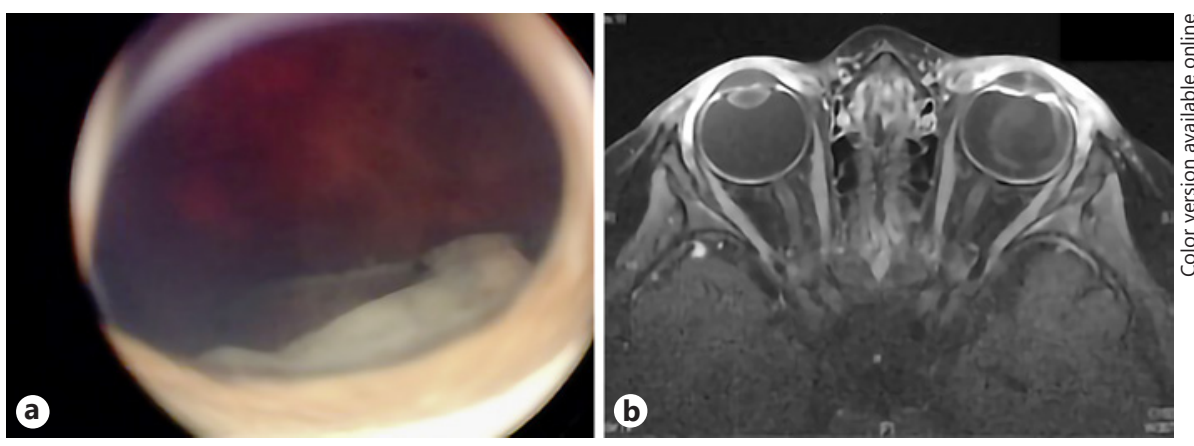

Fig. 3. Fundus photography and corresponding MRI scan of patient No. 8. Note the whitish anteriorly located tumour which could not clearly be classified as a retinoblastoma. the remaining eyes were classified as group $\mathrm{E}$, and of the remaining 2 eyes, one was a group $B$ and the other one a group D eye.

All patients underwent an intensive clinical workup with magnetic resonance imaging (MRI) of the eye, brain and spinal cord if necessary as well as lumbar puncture and bone marrow aspiration. In 1 child, bone marrow infiltration by retinoblastoma was detectable at the time of referral.

The remaining eyes in all but one patient were enucleated immediately, and a detailed histopathological workup on risk factors for metastatic spread and infiltration of the sclerotomy sites was performed on the specimens. The histopathological workup showed optic nerve infiltration in 4 cases, choroidal infiltration in 6 cases, scleral infiltration in 2 eyes, and 1 eye had an extensive extraocular extension. Figure 1 shows the MRI scan with the corresponding histopathological slides and photography of the opened globe with an extensive optic nerve infiltration of at least $5 \mathrm{~mm}$.

Figure 2 shows the MRI scan of patient No. 9 who was treated with a $25-\mathrm{G}$ vitrectomy and silicone oil tamponade due to presumed uveitis. Note the extraocular tumour extension nasal to the optic nerve. 
Table 2. Histopathology, treatment, follow-up and survival

\begin{tabular}{cllllll}
\hline $\begin{array}{l}\text { Patient } \\
\text { No. }\end{array}$ & $\begin{array}{l}\text { ICRB } \\
\text { group }\end{array}$ & $\begin{array}{l}\text { Histopathological } \\
\text { risk factors }\end{array}$ & $\begin{array}{l}\text { Systemic } \\
\text { chemotherapy }\end{array}$ & $\begin{array}{l}\text { Radiation } \\
\text { therapy }\end{array}$ & $\begin{array}{l}\text { Follow-up, } \\
\text { months }\end{array}$ & $\begin{array}{l}\text { Status at } \\
\text { final } \\
\text { follow-up }\end{array}$ \\
\hline 1 & E & ONI, CI & 6 cycles & PB & 55 & AF \\
2 & E & none & 6 cycles & no & 50 & AF \\
3 & E & CI & 6 cycles & EBRT & 147 & AF \\
4 & E & none & 4 cycles & EBRT & 74 & AF \\
5 & E & CI & 4 cycles & IS & 250 & AF \\
6 & D & none & 6 cycles & EBRT & 15 & AF \\
7 & E & ONI, CI, SI & 6 cycles & Ru & 12 & AF \\
8 & B & not enucleated & 6 cycles & IS & 11 & AM \\
9 & E & ONI, CI, OI & 6 cycles & no & 18 & AF \\
\hline
\end{tabular}

ICRB, International Classification of Retinoblastoma; ONI, optic nerve infiltration; CI, choroidal infiltration; SI, scleral infiltration; OI, orbital involvement; PB, proton beam radiation; EBRT, external beam radiation therapy; IS, iodine seeds; Ru, ruthenium plaque; AF, alive, free of metastasis; AM, alive with metastasis. ${ }^{1}$ Refused by parents. ${ }^{2} 25-\mathrm{G}$ system used with free sclerotomy sites.

Evaluating the sclerotomy sites, we found tumour cell distribution in 3 eyes; in all of these cases, a 20-G system was used. In patients treated with a $25-\mathrm{G}$ system, we found no signs of tumour cell invasion along the sclerotomy tract.

In 1 case, initially misdiagnosed with congenital cataract, the differential diagnosis between a group B retinoblastoma and a malignant medulloepithelioma could not be eventually established on the specimens available, even though the externally obtained biopsy samples were analysed by at least 3 experienced pathologists. This was the only child where we decided to postpone secondary enucleation. Figure 3 shows the anteriorly located tumour after cataract surgery.

Adjuvant treatment after enucleation consisted of 4-6 cycles of systemic chemotherapy in all patients. Nine children were treated with a cocktail of vincristine, cyclophosphamide, carboplatin and etoposide. In 1 patient, there was a more aggressive treatment regimen consisting of vincristine, adriablastin, ifosfamide and dacarbazine alternating with vindesine, cisplatin and etoposide. This was 1 of 2 children with a latency of 2 months between initial vitrectomy and referral to our institution. All but 2 children had an adjuvant radiotherapy that consisted of a localized fractionated external beam radiation therapy of the orbit with a cumulative dose of $50 \mathrm{~Gy}$ in 3 cases. Two patients were treated with proton beam again with a total dose of $50 \mathrm{~Gy}$. Two had an afterloading of the orbit with iodine seeds, leading to a total dose of $40 \mathrm{~Gy}$. These were the first children in the series treated at our institution. However, dur- ing follow-up, they showed a corresponding mid-face hypoplasia and the necessity of an implant change and reconstruction of the conjunctival fornices. Therefore, in the further course, other irradiation techniques were preferred. Additional radiation therapy was performed in particular to avoid local recurrences. The child with the unclear diagnosis of retinoblastoma versus medulloepithelioma had a ruthenium plaque at the tumour site with a dose of $88 \mathrm{~Gy}$ at tumour apex. Two patients did not receive an additional radiation therapy. In 1 case, the parents refused the therapy, and in the most recent case, we postponed adjuvant radiotherapy as the child was treated with a selfsealing $25-\mathrm{G}$ system with no signs of histopathological risk factors or tumour cell dissemination at the sclerotomy sites. After extensive discussion with the parents and due to the potential hazards of an additional radiation therapy, we decided to treat the child with systemic chemotherapy alone. This child is under very intense repetitive MRI scan surveillance and has not developed any signs of orbital recurrence or distant metastasis over the last 2 years. Concerning radiation-induced complications after adjuvant radiation therapy, 1 child treated with iodine seeds developed a significant mid-face hypoplasia resulting in severe psychological and cosmetic problems.

One of our patients already had bone marrow metastases on initial presentation and is on high-dose chemotherapy. All other patients were free of metastases or orbital recurrence on the last follow-up, ranging from 11 months to more than 20 years (Table 2 ). 


\section{Discussion}

Retinoblastoma is a rare infant disease that usually presents before 5 years of age [11]. 75\% of unilaterally affected children are diagnosed in their second or third year of life [12]. Typical presenting symptoms for retinoblastoma are leukocoria, followed by strabism. With clear media, the diagnosis can be made easily with standard clinical procedures, including ophthalmoscopy, ultrasound or MRI. Nevertheless, retinoblastoma can sometimes be misdiagnosed when there is opaque media or an atypical presentation of the disease [13].

Shields et al. [14] published their data on 604 cases of so-called pseudo retinoblastomas and found the most common diagnoses to be Coats disease, persistent fetal vasculature, ocular toxocariasis and vitreous haemorrhage. We also analysed 31 cases of advanced stages of unilateral undetermined ocular tumours in childhood treated with enucleation and found the main diagnoses to be Coats disease, astrocytoma, medulloepithelioma or an undetected atypical retinoblastoma [15]. A specific form of the atypical tumour is the so-called diffuse infiltrating retinoblastoma, which is a very rare subtype of this neoplasm and is characterized by its atypical growth pattern. Therefore, this type of tumour may mimic other more innocuous diseases and may be misdiagnosed [16].

It is well known that a delay in treatment, with the risk of tumour cells invading the CNS, regional lymph nodes, bones or bone marrow, or mismanagement of retinoblastoma eyes worsens the prognosis of the young patients considerably [10]. In the presence of metastatic disease or tumour cell spread to the CNS, prognosis is poor, and most patients die of disease progression despite aggressive treatment [17].

One main iatrogenic risk factor for tumour cell spread is a biopsy or vitrectomy in undetected retinoblastoma eyes, as it can lead to orbital recurrence or distant metastases with a poor prognosis [18]. In 2000, Shields et al. [9] analysed 11 patients treated with vitrectomy in an unsuspected retinoblastoma eye. The main preoperative diagnoses in this series were vitreous haemorrhage (64\%), toxocariasis (18\%), toxoplasmosis (9\%) and endophthalmitis (9\%). Age at surgery ranged from 3 to 16 years. All children were enucleated, and there was adjuvant treatment in 10 patients, consisting of orbital radiotherapy, systemic chemotherapy or both. One child was suffering from metastatic disease on referral, the others were well and free of metastases with a mean follow-up of 7 years. A similar study was presented in 2015 by Shen et al. [10] where they published a review of 3 cases of pars plana vit- rectomy and evisceration in misdiagnosed retinoblastoma eyes. Again, the children were older than expected for unilateral retinoblastoma with 4.3 years on average. The most frequent misdiagnoses were Coats disease, ocular blunt trauma and endophthalmitis. In this series, 1 child was treated with systemic chemotherapy and adjuvant radiation therapy, 1 child with adjuvant radiotherapy alone, and in the last child, the parents refused treatment. All 3 patients developed massive orbital extension and died as a result of tumour metastases after a time interval of several months up to 2 years. In a recent paper, Kaliki et al. [19] reported on a series of 14 cases of unsuspected retinoblastoma with inadvertent intraocular surgery. This series focuses on Indian patients where retinoblastoma stages, as a rule, are more advanced and time to referral for retinoblastoma treatment is usually longer, with 7 months on average in this series. Consequently, only 3 of the patients were presenting without extraocular extension and, therefore, survival rates in this series were very poor with metastatic death in $57 \%$ of the patients despite high-dose systemic chemotherapy and maximal surgical treatment, including enucleation or exenteration of the orbit combined with external orbital radiation. Death occurred after several months up to 2 years after correct diagnosis.

In our series of 10 eyes with undetected retinoblastoma, the mean age was slightly lower than in the other series. Again, as in the other series, all patients were suffering from unilateral disease. The most frequent misdiagnoses were unilateral inflammation with a suspected lymphoma, uveitis or toxocariasis in 5 cases (50\%), vitreous haemorrhage after ocular trauma in 2 cases $(20 \%)$, and the last 3 children were misdiagnosed with Coats disease, retinal detachment and congenital cataract $(10 \%$ each). All but one patient were treated with enucleation and with an aggressive treatment consisting of systemic chemotherapy and adjuvant radiation therapy in 8 cases. One patient was suffering from bone marrow metastasis by the time of referral to our hospital. Fortunately, all other patients survived with no evidence of local or distant metastases with a mean follow-up of 5.4 years, which indicates that they are cured as metastases usually arise after 12-15 months [9]. In our series, we postponed additional radiotherapy in 1 child treated with a self-sealing $25-\mathrm{G}$ system, although there is common consent that radiation therapy is essential to achieve local control in patients with orbital involvement of retinoblastoma [17]. As there was no evidence of tumour cell dissemination to the sclerotomy sites, we decided to perform close control followup with MRI scans instead, as it is known that radiation 
therapy can result in severe cosmetic problems due to mid-face hypoplasia in patients treated with enucleation and combined radiation therapy [20].

Fine-needle aspiration biopsy in undetermined ocular tumours in childhood may be another source for leakage of tumour cells to the orbit. In a series published by Karcioglu [8] in 2002, the long-term follow-up of unsuspected retinoblastoma eyes treated with fine-needle aspiration biopsy was evaluated. Five of the 6 affected children were enucleated without additional treatment, while 1 was treated with cryotherapy and plaque placement in the same session. None of the children developed orbital recurrence or distant metastases over a mean follow-up of 10.8 years on average, which is consistent with the findings of our study. Our child still shows no signs of orbital recurrence over a period of 2 years.

Systemic chemotherapy and adjuvant radiotherapy improve the prognosis of retinoblastoma patients with inadvertent surgery considerably. Although the clinical series of these patients (luckily) are small, a less aggressive therapy worsens the prognosis considerably. When taking a closer look at the published data on vitrectomy in undetected retinoblastoma eyes concerning the risk factors of orbital involvement and distant metastases without prompt aggressive treatment, there is a paper by Stevenson et al. [18] published in 1989. They report about a series of 3 children with vitrectomy in undetected retinoblastoma eyes none of which had received any adjuvant treatment to reduce the risk of local or distant metastases. Unfortunately, all of them developed orbital or lymph node metastases 3-6 months after initial treatment. This underlines the urgent need for aggressive treatment in inadvertently vitrectomized retinoblastoma eyes to prevent orbital recurrence or distant metastases.

In summary, we want to emphasize that retinoblastoma can present in an atypical fashion and the differential diagnosis should be kept in mind when older children present with unilateral leukocoria, as the clinical diagnosis with non-invasive methods may be extremely crucial. Liquid biopsy, which is on the horizon of diagnostic modalities, may help resolve this problem. Ghiam et al. [21] could show that tumour-derived cfDNA is present in the aqueous humour and that genomic analyses reflect somatic copy number alteration profiles of the corresponding tumour. They concluded that a liquid biopsy of aqueous humour can be extracted safely via the insertion of a small-gauge needle through the clear cornea, which on analysis can even help to identify the $6 \mathrm{p}$ tumour biomarker gain as an indicator of aggressive disease. In the case of undetected retinoblasto$\mathrm{ma}$, a minimally invasive intervention could, in the case of unclear clinical findings, confirm or exclude the diagnosis of a retinoblastoma accordingly. Nevertheless, even with this new diagnostic tool, one always needs to be aware of the underlying possibility of a retinoblastoma, and if there is uncertainty, consultation of an experienced retinoblastoma reference centre is advised. If vitrectomy has been performed in these patients, consequent and aggressive treatment improves the otherwise bad prognosis considerably and should be initiated without delay.

\section{Statement of Ethics}

All authors confirm that the appropriate ethics review has been followed and the research was done in accordance with the World Medical Association. Since this is an anonymised retrospective work with a long follow-up, a written informed patient consent was not required.

\section{Disclosure Statement}

The authors have no conflicts of interest to declare.

\section{Funding Sources}

This research received no specific grant from any agency in the public, commercial or not-for-profit sectors.

\section{Author Contributions}

Conceptualisation: E.B., N.B., N.E.B.; investigation: E.B., S.S., K.A.M., T.K., P.R., S.G., P.K., D.G.; methodology: E.B., N.B., K.A.M.; writing the original draft: E.B., N.B., N.E.B.; revision for intellectual content: E.B., S.S., N.E.B., T.K., P.R., D.G., K.M., S.G., P.K., N.B. All authors are responsible for the conception of the paper, the data acquisition, analysis and interpretation of data and revised it critically for important intellectual content. All authors gave the final approval of the version to be published. Finally, there is an agreement to be accountable for all aspects of the work in ensuring that questions related to the accuracy or integrity of any part of the work are appropriately investigated and resolved. There are no further non-author contributors.

References

Ocul Oncol Pathol 2020;6:244-250

1 Dimaras H, Corson TW, Cobrinik D, White A, Zhao J, Munier FL, et al. Retinoblastoma. Nat Rev Dis Primers. 2015 Aug; 1(1): 15021.

2 Broaddus E, Topham A, Singh AD. Survival with retinoblastoma in the USA: 1975-2004. Br J Ophthalmol. 2009 Jan;93(1):24-7.

3 Finger PT, Harbour JW, Karcioglu ZA. Risk factors for metastasis in retinoblastoma. Sury Ophthalmol. 2002 Jan-Feb;47(1):1-16. 
4 Gündüz K, Müftüoglu O, Günalp I, Unal E, Taçyildiz N. Metastatic retinoblastoma clinical features, treatment, and prognosis. Ophthalmology. 2006 Sep;113(9):1558-66.

$5 \mathrm{Hu}$ HM, Zhang WL, Wang YZ, Shi JT, Li B, Zhang Y, et al. [Clinical features, treatment and prognosis of retinoblastoma in distant metastasis stage]. Zhonghua Yan Ke Za Zhi. $2017 \mathrm{Feb} ; 53(2): 121-7$.

6 Kopelman JE, McLean IW, Rosenberg SH. Multivariate analysis of risk factors for metastasis in retinoblastoma treated by enucleation. Ophthalmology. 1987 Apr;94(4):371-7.

$7 \mathrm{Hu} \mathrm{H}$, Zhang W, Wang Y, Huang D, Shi J, Li $\mathrm{B}$, et al. Characterization, treatment and prognosis of retinoblastoma with central nervous system metastasis. BMC Ophthalmol. 2018 Apr;18(1):107.

8 Karcioglu ZA. Fine needle aspiration biopsy (FNAB) for retinoblastoma. Retina. 2002 Dec; 22(6):707-10.

9 Shields CL, Honavar S, Shields JA, Demirci H, Meadows AT. Vitrectomy in eyes with unsuspected retinoblastoma. Ophthalmology. 2000 Dec;107(12):2250-5.
10 Shen T, Liu R, Lin J, Huang H, Li X, Yan J. Pars Plana Vitrectomy and Evisceration Resulting in Death Due to Misdiagnosis of Retinoblastoma in Children: A Review of 3 Cases. Medicine (Baltimore). 2015 Aug;94(32): e1338.

11 Aerts I, Lumbroso-Le Rouic L, Gauthier-Villars M, Brisse H, Doz F, Desjardins L. Retinoblastoma. Orphanet J Rare Dis. 2006 Aug; $1(1): 31$.

12 Ortiz MV, Dunkel IJ. Retinoblastoma. J Child Neurol. 2016 Feb;31(2):227-36.

13 Balmer A, Munier F. Differential diagnosis of leukocoria and strabismus, first presenting signs of retinoblastoma. Clin Ophthalmol. 2007 Dec;1(4):431-9.

14 Shields CL, Schoenberg E, Kocher K, Shukla SY, Kaliki S, Shields JA. Lesions simulating retinoblastoma (pseudoretinoblastoma) in 604 cases: results based on age at presentation. Ophthalmology. $2013 \mathrm{Feb} ; 120(2): 311-6$.

15 Schlueter S, Metz K, Bornfeld N, Göricke S, Schlamann M, Sirin S, et al. [Intraocular Tumors other than Retinoblastoma in Children]. Klin Monbl Augenheilkd. 2015 Jul; 232(7):838-44.
16 Traine PG, Schedler KJ, Rodrigues EB. Clinical Presentation and Genetic Paradigm of Diffuse Infiltrating Retinoblastoma: A Review. Ocul Oncol Pathol. 2016 Apr;2(3):12832.

17 Antoneli CB, Steinhorst F, de Cássia Braga Ribeiro K, Novaes PE, Chojniak MM, Arias V, et al. Extraocular retinoblastoma: a 13-year experience. Cancer. 2003 Sep;98(6):1292-8.

18 Stevenson KE, Hungerford J, Garner A. Local extraocular extension of retinoblastoma following intraocular surgery. Br J Ophthalmol. 1989 Sep;73(9):739-42.

19 Kaliki S, Taneja S, Palkonda VAR. Inadvertent intraocular surgery in children with unsuspected retinoblastoma: A study of 14 cases. Retina. 2019 Sep;39(9):1794-1801.

20 Messmer EP, Fritze H, Mohr C, Heinrich T, Sauerwein W, Havers W, et al. Long-term treatment effects in patients with bilateral retinoblastoma: ocular and mid-facial findings. Graefes Arch Clin Exp Ophthalmol. 1991; 229(4):309-14.

21 Ghiam BK, Xu L, Berry JL. Aqueous Humor Markers in Retinoblastoma, a Review. Transl Vis Sci Technol. 2019 Apr;8(2):13. 